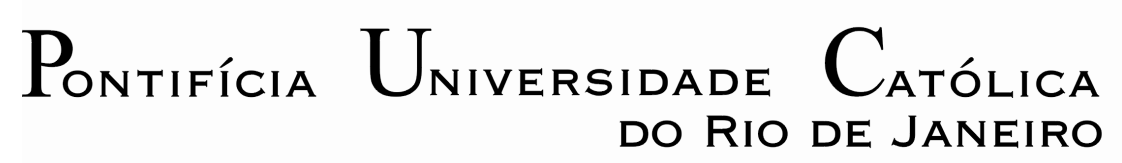

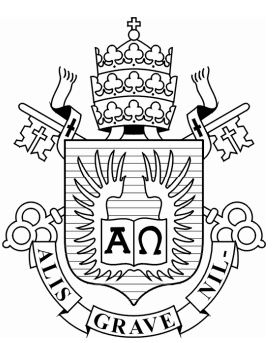

Frederico Martins Alves da Silva

\title{
Modelos de Dimensão Reduzida para Análise das Oscilações Não-Lineares e Estabilidade de Cascas
}

Cilíndricas

Tese de Doutorado

Tese apresentada como requisito parcial para obtenção do título de Doutor pelo Programa de Pós-Graduação em Engenharia Civil da PUC-Rio.

Orientadores: Paulo Batista Gonçalves Zenón José Guzmán Nuñez Del Prado

Rio de Janeiro Março de 2008 


\section{Pontifícia Universidade Católica \\ DO RIO DE JANEIRO}

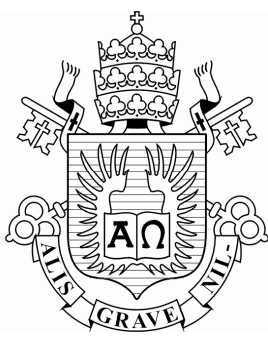

Frederico Martins Alves da Silva

\section{Modelos de Dimensão Reduzida para Análise das \\ Oscilações Não-Lineares e Estabilidade de Cascas}

Cilíndricas

Tese apresentada como requisito parcial para obtenção do título de Doutor pelo Programa de Pós-Graduação em Engenharia Civil da PUC-Rio. Aprovada pela Comissão Examinadora abaixo assinada.

Prof. Paulo Batista Gonçalves

Presidente/Orientador Departamento de Engenharia Civil - PUC-Rio

Prof. Zenón José Guzmán Nuñez Del Prado Co-Orientador Escola de Engenharia Civil - UFG

Prof. Carlos Eduardo Nigro Mazzilli USP - SP

Prof. Ronaldo Carvalho Battista COPPE - UFRJ

Prof. João Luís Pascal Roehl PUC-Rio

Prof. Raul Rosas e Silva PUC-Rio

Prof. José Eugênio Leal

Coordenador(a) Setorial do Centro Técnico Científico - PUC-Rio 
Todos os direitos reservados. É proibida a reprodução total ou parcial do trabalho sem autorização da universidade, do autor e do orientador.

\section{Frederico Martins Alves da Silva}

Graduou-se em Engenharia Civil pela Universidade Católica de Goiás (UCG) em janeiro de 2002. Ingressou no mestrado em Engenharia Civil da Universidade Federal de Goiás (UFG) em março de 2002, atuando na área de Instabilidade e Dinâmica das Estruturas. Em 2004, continuando na mesma linha de pesquisa do mestrado, iniciou o curso de doutorado na PUC-Rio.

Ficha Catalográfica

Silva, Frederico Martins Alves da

Modelos de dimensão reduzida para análise das oscilações não-lineares e estabilidade de cascas cilíndricas / Frederico Martins Alves da Silva ; orientadores: Paulo Batista Gonçalves, Zenón José Guzmán Nuñez Del Prado. - 2008.

182 f. : il. (col.) ; $30 \mathrm{~cm}$

Tese (Doutorado em Engenharia Civil)-Pontifícia Universidade Católica do Rio de Janeiro, Rio de Janeiro, 2008.

Inclui bibliografia

1. Engenharia civil - Teses. 2. Cascas cilíndricas. 3. Modelos de dimensão reduzida. 4. Método de KarhunenLoève. 5. Interação fluido-estrutura. 6. Oscilações nãolineares. 7. Fator de integridade. 8. Estabilidade dinâmica. I. Gonçalves, Paulo Batista. II. Prado, Zenón José Guzmán Nuñez Del. III. Pontifícia Universidade Católica do Rio de Janeiro. Departamento de Engenharia Civil. IV. Título. 


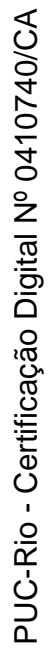

À Renata, à Elizabeth e aos familiares com amor e carinho. 


\title{
Agradecimentos
}

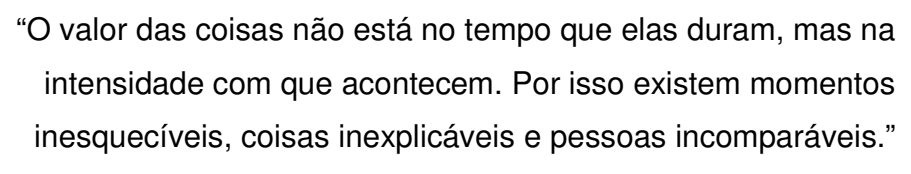

(Fernando Pessoa).

\begin{abstract}
À Deus pela Sua infinita bondade claramente manifestada nos professores que tive ao longo de toda vida, acadêmicos ou não, sempre encontrei pessoas que tinham algo de bom a ensinar.
\end{abstract}

Aos meus familiares. Peço licença para citá-los: Elizabeth, Bráulio, Margaret, Jorge, Cirlei, Braulin, Elizângela, Cristiane, Cecília, Guto, Yana, Lídia, Nathália, Ana Cristina, João, Otília, Milton, André Luiz, Fernanda, Alessandra, Roberto, Maria Cecília e, nesse mistério que compõe a vida, ao mais novo membro da família, que para ciência ainda não é possível sabermos se será uma menina ou menino, mas desde já deixo registrado o meu amor. Cada um de vocês e a cada dia fazem parte do meu eterno aprendizado. Sem falar do meu eterno amor, Renata, que me faz uma pessoa melhor a cada instante desse caminho chamado felicidade.

Ao Prof. Paulo B. Gonçalves, que com toda sua paciência, dedicação, amizade e solicitude, ensinou-me muito mais do que os livros lidos e a pesquisa desenvolvida. Sim importantes! Mas mostrou-me que semear tais virtudes é o único caminho para tornar-se educador.

Ao dileto amigo Zenón J. G. N. Del Prado. Sei que seu papel na concretização dessa etapa é tão importante quanto de quem a trilhou. Sei também, que tudo o que já passamos em torno das cascas, o que fica é a nossa amizade e companheirismo. Você é com certeza um daqueles irmãos que ao longo da vida aparecem e, sem que se perceba, tornam-se inesquecíveis.

À Prof. Sylvia Regina Mesquita de Almeida e ao Prof. Ademir Aparecido do Prado, ambos da UFG. Seus ensinamentos no mestrado diminuíram os obstáculos desta jornada.

Aos amigos de república que me aturaram e que suportaram as minhas manias: Pasquetti, José Roberto, Diego, Walter, Magnus e Roberto. 
Aos amigos da sala 609 que propiciaram ao longo desses anos uma formação extracurricular em nossos calorosos debates. Bastava que alguma notícia fosse publicada e lá estávamos analisando-a, como se detivéssemos todo o conhecimento do mudo para tal. "Não concordo com uma única palavra do que dizeis, mas defenderei até a morte o vosso direito de dizê-la." (Voltaire). A esses amigos: Müller, Diego, Igor, Pecin e João meu fraterno abraço.

Ao Prof. Carlos A. Almeida e a Prof. Angela F. Perricone pelo exemplo do magistério.

Aos Profs. M. Amabili, G. Rega e S. Lenci pela prestatividade concedida na análise dos resultados desta tese.

Aos demais professores do departamento de Engenharia Civil.

A Capes e ao CNPq pelo apoio financeiro, sem os quais este trabalho não poderia ser realizado. 


\section{Resumo}

Silva, Frederico Martins Alves da; Gonçalves, Paulo Batista; Prado, Zenón José Guzmán Nuñez Del. Modelos de dimensão reduzida para análise das oscilações não-lineares e estabilidade de cascas cilíndrica. Rio de Janeiro, 2008. 182p. Tese de Doutorado - Departamento de Engenharia Civil, Pontifícia Universidade Católica do Rio de Janeiro.

Nesta tese, as vibrações não-lineares e a estabilidade de uma casca cilíndrica contendo um fluido são estudadas com base em modelos de dimensão reduzida, isto é, modelos com um número reduzido de graus de liberdade. A partir dos funcionais de energia potencial e cinética de uma casca cilíndrica, deduzem-se suas equações de movimento. O campo de deformações da casca cilíndrica segue a teoria não-linear de Donnell para cascas abatidas. O fluido é considerado interno à casca irrotacional, não-viscoso e incompressível, sendo descrito a partir de um potencial de velocidade que leva em consideração a interação entre o fluido e a estrutura. Para resolver o sistema de equações de equilíbrio da casca, desenvolve-se um procedimento analítico que permite obter os campos de deslocamento axial e circunferencial em função dos deslocamentos laterais, além de atender as condições de contorno do problema. Desta forma, reduz-se o sistema de equações de equilíbrio a uma única equação diferencial parcial que é resolvida com o método de Galerkin. A determinação dos deslocamentos laterais é feita a partir de técnicas de perturbação que ordena os modos não-lineares de acordo com sua importância na solução da casca cilíndrica. Comprova-se essa ordenação através do método de KarhunenLoève que fornece, também, uma expansão ótima para os deslocamentos laterais. Além dessas técnicas, apresenta-se uma redução polinomial que relacionam as amplitudes dos modos não-lineares com a amplitude do modo linear, criando uma expansão modal com 1 GDL. Apresentam-se respostas no tempo, fronteiras de instabilidade e diagramas de bifurcação para uma casca cilíndrica submetida a dois tipos de carregamentos harmônicos, pressão lateral e carga axial. A seguir, são propostos alguns critérios para a análise da a integridade do sistema dinâmico tanto para um sistema com 1 GDL quanto para um sistema multidimensional através da evolução e erosão das bacias de atração. Por fim, estuda-se o comportamento de cascas cilíndricas parcialmente cheias, mostrando a influência da altura do fluido nas fronteiras de instabilidade e curvas de ressonância da casca cilíndrica.

\section{Palavras-chave}

cascas cilíndricas; modelos de dimensão reduzida; método de KarhunenLoève; interação fluido-estrutura; oscilações não-lineares; fator de integridade; estabilidade dinâmica. 


\section{Abstract}

Silva, Frederico Martins Alves da; Gonçalves, Paulo Batista (Advisor); Prado, Zenón José Guzmán Nuñez Del (Co-advisor). Low dimensional models for nonlinear vibration analysis and stability of cylindrical shells. Rio de Janeiro, 2008. 182p. Tese de Doutorado - Departamento de Engenharia Civil, Pontifícia Universidade Católica do Rio de Janeiro.

The nonlinear vibrations and stability of a fluid-filled cylindrical shell is investigated using reduced order models. First, the nonlinear equations of motion of the cylindrical shell are deduced based on the expressions for the potential and kinetic energy, which are obtained using Donnell shallow shell theory. The internal fluid is considered to be irrotational, non-viscous and incompressible. It is described by a velocity potential that takes into account the fluid-shell interaction. A procedure is proposed to obtain analytically the axial and circumferential displacements of the shell, satisfying the in-plane equations of motion and the associated boundary conditions. So, the problem is reduced to one partial differential equation of motion which is solved by the Galerkin method. The transversal displacement field is obtained by perturbation techniques. This enables one to identify the relevance of each term in the nonlinear expansion of the vibration modes. Then, the Karhunen-Loève method is employed to investigate de relative importance of each mode obtained by the perturbation analysis on the nonlinear response and to deduce optimal interpolation function to be used in the Galerkin procedure. A SDOF model is also obtained by relating the modal amplitudes of the nonlinear modes to the vibration amplitude of the linear mode. Time responses, instability boundaries and bifurcation diagrams are obtained for cylindrical shells subjected to harmonic lateral and axial loads. Different procedures for the analysis of the shell integrity are proposed based on the evolution and erosion of the basins of attraction in state-space. Finally, the influence of the fluid height on the stability boundaries and resonance curves is studied.

\section{Keywords}

cylindrical shells, reduced order models, Karhunen-Loève method, fluidstructure interaction, nonlinear oscillations, integrity factor, dynamic stability 


\section{Sumário}

1 INTRODUÇÃO

1.1. MOTIVAÇÃO

1.2. OBJETIVOS 31

1.3. ORGANIZAÇÃO DO TRABALHO 32

2 MODELAGEM DA CASCA CILÍNDRICA

2.1. CAMPO DE DEFORMAÇÕES 34

2.2. ESFORÇOS DE MEMBRANA E DE FLEXÃO 36

2.3. FUNCIONAIS DE ENERGIA DA CASCA CILÍNDRICA 37

2.4. SISTEMA DE EQUAÇÕES NÃO-LINEARES 39

2.5. SISTEMA DE EQUAÇÕES NÃO-LINEARES ADIMENSIONAIS 42

3 MODELAGEM DO FLUIDO INTERNO 44

3.1. EQUAÇÕES BÁSICAS DO FLUIDO 44

3.2. DETERMINAÇÃO DO POTENCIAL DE VELOCIDADE DO FLUIDO 46

3.3. CONSIDERAÇÕES SOBRE A VIBRAÇÃO DA SUPERFÍCIE LIVRE DO FLUIDO

(SLOSHING) 50

4 MODELOS REDUZIDOS PARA A CASCA CILÍNDRICA 52

4.1. SOLUÇÃO GERAL DO CAMPO DE DESLOCAMENTO DA CASCA PELO MÉTODO DA PERTURBAÇÃO 53

4.2. DETERMINAÇÃO DO CAMPO DE DESLOCAMENTOS UE $V$

4.3. REDUÇÃO dO PROBLEMA PELO MÉTOdO DE KARHUNEN-LOĖVE

4.4. OBTENÇÃO DOS MODELOS REDUZIDOS PARA CASCAS CILÍNDRICAS 61

4.4.1. Análise estática não-linear 62

4.4.2. Análise dinâmica não-linear 68

4.5. MODELO REDUZIDO VERSUS MÉTODO DOS ELEMENTOS FINITOS

5 ANÁLISE DAS VIBRAÇÕES NÃO-LINEARES

5.1. CASCA CILÍNDRICA, SIMPLESMENTE APOIADA, SUBMETIDA A UMA PRESSÃO LATERAL HARMÔNICA

5.1.1. Influência do pré-carregamento axial, $\Gamma_{0}$. 86

5.1.2. Modelo reduzido versus Método dos elementos finitos 89

5.2. CASCA CILÍNDRICA, SIMPLESMENTE APOIADA, SUBMETIDA A UMA CARGA AXIAL HARMÔNICA 92

6 INTEGRIDADE DE SISTEMAS NÃO-LINEARES

6.1. INTEGRIDADE DO SISTEMA DINÂMICO COM UM GRAU DE LIBERDADE 112

6.1.1. Vibração livre não-amortecida 112 
6.1.2. Vibração livre amortecida 114

6.1.3. Vibração forçada amortecida $\quad 119$

6.2. INTEGRIDADE DE UM SISTEMA DINÂMICO MULTIDIMENSIONAL 130

6.3. CASCA CILÍNDRICA COMPLETAMENTE CHEIA 146

6.3.1. Modelo com um grau de liberdade 146

6.3.2. Modelo multidimensional 152

7 CASCA CILÍNDRICA PARCIALMENTE CHEIA 159

8 CONCLUSÕES E SUGESTÕES 168

8.1. CONCLUSÕES 168

8.2. SUGESTÕES 170

9 REFERÊNCIAS BIBLIOGRÁFICAS 171

$\begin{array}{ll}\text { APÊNDICE A } & 178\end{array}$ 


\section{Lista de Figuras}

Figura 1.1 - Torre de resfriamento de Niederaussem - Alemanha (Busch et al., 2002).

Figura 2.1 - Geometria e campo de deslocamentos da casca cilíndrica.

Figura 2.2 - Convenção de sinais e resultante dos esforços (a) de membrana e (b) de flexão

Figura 2.3 - Representação do carregamento aplicado à casca. .................................... 37

Figura 3.1 - Representação esquemática do fluido interno à casca cilíndrica..................4 47

Figura 4.1 - Caminho pós-critico da casca cilíndrica, considerando diferentes expansões para os deslocamentos radiais.

Figura 4.2 - Caminho pós-critico da casca cilíndrica. Comparação entre a formulação por função de tensão e a formulação com $u$ e $v$ em função de $w$.

Figura 4.3 - Quatro primeiros POMs com os seus respectivos POVs para a resposta não-linear pós-crítica.

Figura 4.4 - Comparação entre a solução pós-crítica da casca a partir da expansão (4.21) com a reconstrução da resposta usando os quatros primeiros POMs.

Figura 4.5 - Comparação do caminho pós-crítico utilizando a expansão modal (4.21) e o modelo com apenas um grau de liberdade (4.34).

Figura 4.6 - Relação freqüência-amplitude para a casca cilíndrica, considerando diferentes expansões para os deslocamentos radiais.

Figura 4.7 - Quatro primeiros POMs e seus respectivos POVs para a vibração livre não-linear da casca cilíndrica.

Figura 4.8 - Relação freqüência-amplitude para a casca cilíndrica usando a expansão modal obtida pelo método da perturbação e pela expansão de KarhunenLoève.

Figura 4.9 - Convergência da relação freqüência-amplitude usando um modelo de dimensão reduzida com o aumento do número de POMs

Figura 4.10 - Variação de cada amplitude modal ao longo de um período de vibração livre. $\Omega=0.98$.

Figura 4.11 - Comparação da relação freqüência-amplitude utilizando a expansão modal (4.21) e o modelo com apenas um grau de liberdade (4.38).

Figura 4.12 - Campo de deslocamentos (a) radiais e (b) axiais da casca cilíndrica para o modo $m=1$ e $n=5$. Resultado obtido através do Abaqus

Figura 4.13 - Variação do deslocamento radial no ponto $(0,2 ; 0)$ em função do tempo obtido a partir do Abaqus.

Figura 4.14 - Comparação entre os resultados do Abaqus e o modelo analítico da relação freqüência-amplitude.

Figura 4.15 - Quatro primeiros POMs e seus respectivos POVs para a vibração livre não-linear e levemente amortecida da casca cilíndrica obtida a partir do Abaqus. 
Figura 5.1 - Máximo deslocamento lateral versus freqüência de excitação para uma casca vazia.

Figura 5.2 - Máximo deslocamento lateral versus freqüência de excitação para uma casca completamente cheia.

Figura 5.3 - Máximo deslocamento lateral versus freqüência de excitação para uma casca vazia e $\Gamma_{2}=0,50$.

Figura 5.4 - Máximo deslocamento lateral versus freqüência de excitação para uma casca completamente cheia e $\Gamma_{2}=0,50$.

Figura 5.5 - Comparação entre os modelos de $1 \mathrm{GDL}, 6 \mathrm{GDL}$ e os resultados do Abaqus para o deslocamento lateral do ponto de coordenada $(x, \theta)=(\mathrm{L} / 2 ; 0)$ de uma casca cilíndrica vazia. $\Gamma_{2}=1,0$.

Figura 5.6 - Bacia de atração para a casca cilíndrica vazia. $\left(\Gamma_{2}=1,00\right)$. Modelo com 1 GDL. $\mathbf{~ - ~ s o l u c ̧ a ̃ o ~ d e ~ p e q u e n a ~ a m p l i t u d e . ~} 1$ - solução de grande amplitude,

Figura 5.7 - Caminho pós-crítico da casca cilíndrica variando a expansão modal para os deslocamentos laterais

Figura 5.8 - Fronteiras de instabilidade paramétrica e de escape para uma casca cilíndrica vazia e outra completamente cheia. Carregamento brusco. $\Gamma_{0}=0,40$. $\left(\Gamma_{0}>\Gamma_{\mathrm{MIN}}-6 \mathrm{GDL}=0,20\right)$.

Figura 5.9 - Fronteiras de instabilidade paramétrica e de escape para uma casca cilíndrica vazia e outra completamente cheia. Carregamento brusco. $\Gamma_{0}=0,60$. $\left(\Gamma_{0}>\Gamma_{\mathrm{MIN}}-6 \mathrm{GDL}=0,20\right)$.

Figura 5.10 - Fronteiras de instabilidade paramétrica e de escape para uma casca cilíndrica vazia e outra completamente cheia. Carregamento brusco. $\Gamma_{0}=0,80$. $\left(\Gamma_{0}>\Gamma_{\mathrm{MIN}}-6 \mathrm{GDL}=0,20\right)$.

Figura 5.11 - Respostas no tempo e planos de fase para uma casca cilíndrica vazia. Modelo reduzido de $6 \mathrm{GDL}$. $\Gamma_{0}=0,40$ e $\Omega=1,60$. - pontos fixos do mapa de Poincaré da resposta permanente.

Figura 5.12 - Respostas no tempo e planos de fase para uma casca cilíndrica completamente cheia. Modelo reduzido de $6 \mathrm{GDL}$. $\Gamma_{0}=0,40$ e $\Omega=0,92$. $\bullet$ - pontos fixos do mapa de Poincaré da resposta permanente.

Figura 5.13 - Fronteiras de instabilidade paramétrica e de escape para uma casca cilíndrica vazia e outra completamente cheia. Carregamento gradual. $\Gamma_{0}=0,40$. $\left(\Gamma_{0}>\Gamma_{\mathrm{MIN}}-6 \mathrm{GDL}=0,20\right)$.

Figura 5.14 - Fronteiras de escape permanente para uma casca cilíndrica vazia e outra completamente cheia. Comparação entre um carregamento gradual e um carregamento brusco. $\Gamma_{0}=0,40 .\left(\Gamma_{0}>\Gamma_{\mathrm{MIN}-6 \mathrm{GDL}}=0,20\right)$.

Figura 5.15 - Fractalidade das fronteiras de escape permanente de uma casca cilíndrica vazia e $\Gamma_{0}=0,40$. Carregamento brusco. 
Figura 5.16 - Fractalidade das fronteiras de escape permanente de uma casca cilíndrica completamente cheia e $\Gamma_{0}=0,40$. Carregamento brusco.

Figura 5.17 - Diagramas de bifurcação para uma casca cilíndrica vazia. $\Gamma_{0}=0,40$.

Região principal de instabilidade paramétrica.

Figura 5.18 - Diagramas de bifurcação para uma casca cilíndrica completamente cheia. $\Gamma_{0}=0,40$. Região principal de instabilidade paramétrica. 105

Figura 5.19 - Diagrama de bifurcação, para uma casca cilíndrica vazia, obtido pelo método da Força Bruta. $\left(\Gamma_{0}=0,40\right)$.

Figura 5.20 - Diagramas de bifurcação para uma casca cilíndrica vazia. Modelo com $6 \mathrm{GDL}$. $\Gamma_{0}=0,40$. Região secundária de instabilidade paramétrica. 108

Figura 5.21 - Planos de fase da região secundária de instabilidade paramétrica da casca cilíndrica vazia. Modelo com 6 GDL. $\left(\Gamma_{0}=0,40\right)$.

Figura 5.22 - Formas como os multiplicadores de Floquet podem sair do círculo de raio unitário.

Figura 6.1 - Caminho pós-crítico da casca cilíndrica. Modelo com 1 GDL.

Figura 6.2 - Órbitas heteroclínicas e homoclínicas da casca cilíndrica, modelo com

1 GDL. $\left(\Gamma_{0}=0,40\right)$.

Figura 6.3 - Plano de fase com diferentes condições iniciais de um sistema conservativo e de um amortecido. $\left(\Gamma_{0}=0,40\right)$.

Figura 6.4 - Bacia de atração estática para um sistema levemente amortecido.

Modelo com $1 \mathrm{GDL}$. $\left(\Gamma_{0}=0,40\right)$.

Figura 6.5 - Instantes iniciais da resposta no tempo para diversas perturbações iniciais.

Figura 6.6 - (a-c) Variação da energia potencial em função de $\Gamma_{0}$ e (d) variação da profundidade dos vales potenciais com o parâmetro $\Gamma_{0}$

Figura 6.7 - Órbitas heteroclínicas e homoclínicas da casca cilíndrica, modelo com 1 GDL. $\Gamma_{0}=0,60$

Figura 6.8 - Variação da área da bacia pré-flambagem com o parâmetro de précarregamento estático.

Figura 6.9 - Diagrama de bifurcação para uma casca cilíndrica vazia. $\left(\Gamma_{0}=0,40\right.$, $\Omega=1,80)$. Modelo com $1 \mathrm{GDL}$

Figura 6.10 - Planos de fase para uma casca cilíndrica vazia. $\left(\Gamma_{0}=0,40, \Omega=1,80\right) \ldots 121$ Figura 6.11 - Bacia de atração permanente para uma casca cilíndrica vazia e fator de integridade. $\left(\Gamma_{0}=0,40, \Omega=1,80\right)$. Modelo com $1 \mathrm{GDL}$.

Figura 6.12 - Variação do fator de integridade bacia de atração da casca cilíndrica vazia. $\left(\Gamma_{0}=0,40, \Omega=1,80\right)$. Modelo com $1 \mathrm{GDL}$.

Figura 6.13 - Relação entre o plano de fase e a coordenada $\zeta_{11}$ do ponto de sela para casca vazia. $\left(\Gamma_{0}=0,40, \Omega=1,80\right)$. Modelo com 1 GDL. $\zeta_{11}(0)=\dot{\zeta}_{11}(0)=1 \times 10^{-4}$. Carga brusca. $\left(\Gamma_{1 \text { CR-BRUSCO }}=1,01 ; \Gamma_{1 \text { CR-GRADUAL }}=1,10\right) \ldots . .124$ 
Figura 6.14 - Superfície de energia total de um sistema conservativo com o plano de fase, Figura $6.13 \mathrm{e}$, de uma casca cilíndrica vazia submetida a um carregamento axial harmônico.

Figura 6.15 - Bacia de atração permanente para uma casca cilíndrica vazia na região de interesse delimitada pelos pontos de sela. $\left(\Gamma_{0}=0,40, \Omega=1,80\right)$. Modelo com 1 GDL. Carregamento brusco.

Figura 6.16 - Bacia de atração transiente para uma casca cilíndrica vazia na região de interesse delimitada pelos pontos de sela. Modelo com $1 \mathrm{GDL}$.

Figura 6.17 - Erosão da área de interesse da bacia de atração para casca vazia.

Modelo com $1 \mathrm{GDL}$. $\left(\Gamma_{0}=0,40, \Omega=1,80\right)$.

Figura 6.18 - Variação do fator de integridade com ângulo de fase. $\left(\Gamma_{0}=0,40, \Gamma_{1}=\right.$ $0,80, \Omega=1,80$ ). Modelo com $1 \mathrm{GDL}$.

Figura 6.19 - (a-c) Projeção do caminho pós-critico e (d) energia potencial total $\Pi$ relativa aos pontos de sela. Modelo com 3 POMs.

Figura 6.20 - (a) Representação, em $\mathfrak{R}^{3}$, da energia potencial total relativa aos pontos de sela e (b) sua intersecção com um plano que passa por ambas as selas e pela origem

Figura 6.21 - Projeções tridimensionais da superfície de energia. $\left(\Gamma_{0}=0,40\right)$.

Figura 6.22 - Projeções da órbita que emerge do ponto de sela na direção do autovetor. $\left(\Gamma_{0}=0,40\right)$.

Figura 6.23 - Secções transversais da energia potencial total, П, (Figura 6.22a).

Figura 6.24 - Diagrama de bifurcação para uma casca cilíndrica vazia. $\left(\Gamma_{0}=0,40\right.$, $\Omega=1,80$ ). Modelo com 3 POMs.

Figura 6.25 - Bacia de atração permanente para uma casca cilíndrica vazia e fator de integridade. ( $\left.\Gamma_{0}=0,40, \Omega=1,80\right)$. Modelo com 3 POMs.

Figura 6.26 - Fluxograma para a determinação do fator de integridade. Modelo com 3 POMs.

Figura 6.27 - Variação do fator de integridade bacia de atração da casca cilíndrica vazia. $\left(\Gamma_{0}=0,40, \Omega=1,80\right)$. Comparação entre os modelos reduzidos com $1 \mathrm{GDL} e$ com 3 POM.

Figura 6.28 - Projeção da resposta permanente no plano $\bar{A}_{1} \times \bar{A}_{2} \cdot\left(\Gamma_{0}=0,40, \Omega=\right.$ 1,80). Modelo com 3 POMs. Carga brusca. $\left(\Gamma_{1 \mathrm{CR}-\mathrm{BR} U \mathrm{sCO}}=1,06 ; \Gamma_{1 \mathrm{CR}-\mathrm{GRADUAL}}=1,13\right)$. $A_{i}(0)=\dot{A}_{i}(0)=1 \times 10^{-4}$

Figura 6.29 - Projeções da resposta permanente da casca em 15 planos distintos.

$\left(\Gamma_{0}=0,40, \Gamma_{1}=1,05, \Omega=1,80\right)$.

Figura 6.30 - Bacia de atração permanente para uma casca cilíndrica vazia na região do interesse do vale pré-flambagem. $\left(\Gamma_{0}=0,40, \Omega=1,80\right)$. Modelo com 3 POMs 
Figura 6.31 - Bacia de atração transiente para uma casca cilíndrica vazia na região de interesse do vale pré-flambagem. $\left(\Gamma_{0}=0,40, \Omega=1,80\right)$. Modelo com 3 POMs. .

Figura 6.32 - Variação do fator de integridade misto da bacia de atração de uma casca vazia. ( $\left.\Gamma_{0}=0,40, \Omega=1,80\right)$. Modelo com 3 POMs.

Figura 6.33 - Variação do fator de integridade de uma casca cilíndrica vazia com ângulo de fase. $\left(\Gamma_{0}=0,40, \Gamma_{1}=0,80, \Omega=1,80\right)$. Comparação entre os modelos reduzidos com $1 \mathrm{GDL}$ e com 3 POM.

Figura 6.34 - Diagrama de bifurcação para uma casca cilíndrica completamente cheia. $\left(\Gamma_{0}=0,40, \Omega=0,90\right)$. Modelo com $1 \mathrm{GDL}$

Figura 6.35 - Bacia de atração permanente para uma casca cilíndrica completamente cheia na região de interesse versus fator de integridade. $\left(\Gamma_{0}=0,40\right.$, $\Omega=0,90$ ). Modelo com $1 \mathrm{GDL}$.

Figura 6.36 - Variação do fator de integridade bacia de atração da casca cilíndrica completamente cheia. $\left(\Gamma_{0}=0,40, \Omega=0,90\right)$. Modelo com $1 \mathrm{GDL}$.

Figura 6.37 - Relação entre o plano de fase e a coordenada $\zeta_{11}$ do ponto de sela para a casca completamente cheia. $\left(\Gamma_{0}=0,40, \Omega=0,90\right) . \quad \zeta_{11}=\dot{\zeta}_{11}=1 \times 10^{-4}$. Carga brusca. $\left(\Gamma_{1 \text { CR-BRUSCO }}=0,75 ; \Gamma_{1 \text { CR-GRADUAL }}=0,96\right)$.

Figura 6.38 - Bacia de atração transiente para uma casca cilíndrica completamente cheia na região de interesse. $\left(\Gamma_{0}=0,40, \Omega=0,90\right)$. Modelo com $1 \mathrm{GDL}$.

Figura 6.39 - Erosão da área da região de interesse da bacia de atração. $\left(\Gamma_{0}=0,40\right.$, $\Omega=0,90)$.

Figura 6.40 - Variação do fator de integridade de uma casca cilíndrica completamente cheia com o ângulo de fase. $\left(\Gamma_{0}=0,40, \Gamma_{1}=0,60, \Omega=0,90\right)$. Modelo com $1 \mathrm{GDL}$.

Figura 6.41 - Diagrama de bifurcação para uma casca cilíndrica completamente cheia. $\left(\Gamma_{0}=0,40, \Omega=0,90\right)$. Modelo com 3 POMs.

Figura 6.42 - Variação do fator de integridade da bacia de atração da casca completamente cheia. $\left(\Gamma_{0}=0,40, \Omega=0,90\right)$. Modelo com 3 POMs.

Figura 6.43 - Projeção da resposta permanente no plano $\bar{A}_{1}(\tau) \times \bar{A}_{2}(\tau)$ para a casca completamente cheia. $\left(\Gamma_{0}=0,40, \Omega=0,90\right)$. Modelo com 3 POMs. $A_{i}=\dot{A}_{i}=1 \times 10^{-4}$. Carga brusca. $\left(\Gamma_{1 \mathrm{CR}-\mathrm{BRUSCO}}=0,84 ; \Gamma_{1 \mathrm{CR}-\mathrm{GRADUAL}}=0,90\right)$

Figura 6.44 - Projeções da resposta permanente da casca completamente cheia. $\left(\Gamma_{0}=0,40, \Gamma_{1}=0,83, \Omega=0,90\right)$.

Figura 6.45 - Bacia de atração permanente para uma casca cilíndrica completamente cheia na região do vale pré-flambagem. $\left(\Gamma_{0}=0,40, \Omega=0,90\right)$. Modelo com 3 POMs.

Figura 6.46 - Bacia de atração transiente para uma casca cilíndrica completamente cheia na região do vale pré-flambagem. $\left(\Gamma_{0}=0,40, \Omega=0,90\right)$. Modelo com 3 POMs... 157 
Figura 6.47 - Variação do fator de integridade misto da bacia de atração para uma casca cilíndrica completamente cheia com $\Gamma_{1}$. $\left(\Gamma_{0}=0,40, \Omega=0,90\right)$. Modelo com 3 POMs

Figura 6.48 - Variação do fator de integridade misto de uma casca cilíndrica completamente cheia com ângulo de fase. $\left(\Gamma_{0}=0,40, \Gamma_{1}=0,60, \Omega=0,90\right)$. Modelo com 3 POMs.

Figura 7.1 - Relação freqüência-amplitude para a casca cilíndrica variando a altura do fluido interno e as considerações sobre o fundo do tanque.

Figura 7.2 - Curvas de ressonância - variação do módulo da amplitude modal $\zeta_{11}$ com a freqüência de excitação para valores crescentes de pressão lateral, $\Gamma_{2}$, e fundo flexível $(\varepsilon=0,001)$.

Figura 7.3 - Curvas de ressonância - variação do módulo da amplitude modal $\zeta_{11}$ com a freqüência de excitação para valores crescentes de pressão lateral, $\Gamma_{2}$, e fundo rígido $(\varepsilon=0,001)$.

Figura 7.4 - Variação das fronteiras de instabilidade da casca cilíndrica, apoiada sobre um fundo flexível, com a altura do fluido interno. Carga brusca. $\left(\Gamma_{0}=0,40\right)$

Figura 7.5 - Variação das fronteiras de instabilidade da casca cilíndrica, apoiada sobre um fundo rígido, com a altura do fluido interno. Carga brusca. $\left(\Gamma_{0}=0,40\right)$ 164

Figura 7.6 - Resposta no tempo e plano de fase para diversos níveis de fluido interno. Fundo flexível. ( $\left.\Gamma_{0}=0,40, \Gamma_{1}=0,60, \Omega=1,05\right)$.

Figura 7.7 - Diagrama de bifurcação em função freqüência da excitação. $\left(\Gamma_{0}=0,40\right.$ e $\left.\Gamma_{1}=0,60\right)$. Modelo com 6 GDL 166

Figura 7.8 - Diagrama de bifurcação em função do nível fluido interno. $\left(\Gamma_{0}=0,40\right.$; $\left.\Gamma_{1}=0,60 ; \Omega=1,05\right)$. Modelo com $6 \mathrm{GDL}$ 


\section{Lista de Tabelas}

Tabela 4.1 - Participação dos modos usados na expansão modal dos quatro primeiros POMs no caminho pós-critico da casca. $\quad 66$

Tabela 4.2 - Participação dos modos usados na expansão modal dos quatro primeiros POMs na relação freqüência-amplitude da casca. $\quad 71$

Tabela 4.3 - Freqüências naturais, em Hz, para uma casca cilíndrica simplesmente apoiada.

Tabela 4.4 - Participação dos modos usados na expansão modal dos quatro primeiros POMs obtidos a partir do método do elementos finitos.

Tabela 5.1 - Máximo deslocamento lateral registrado nas Figuras 5.1 e 5.2.

Tabela 5.2 - Freqüência natural da casca cilíndrica variando o pré-carregamento estático, $\Gamma_{0}$, para uma casca cilíndrica vazia e outra completamente cheia.

Tabela 5.3 - Multiplicador de Floquet crítico para o diagrama de bifurcação da Figura 5.17l. $\left(\Gamma_{0}=0,40\right.$ e $\left.\Omega=1,80\right)$.

Tabela 5.4 - Multiplicador de Floquet crítico para o diagrama de bifurcação da Figura 5.20d. $\left(\Gamma_{0}=0,40\right.$ e $\left.\Omega=0,80\right)$.

Tabela 6.1 - Pontos de equilíbrio e seus respectivos autovalores. Modelo reduzido com 1 GDL. $\Gamma_{0}=0,40$.

Tabela 6.2 - Autovalores reais da sela e seus respectivos autovetores. $\left(\Gamma_{0}=0,40\right) \quad 114$ Tabela 6.3 - Pontos de equilíbrio e seus respectivos autovalores. Modelo reduzido com $1 \mathrm{GDL}$. $\left(\Gamma_{0}=0,60\right)$.

Tabela 6.4 - Pontos de equilíbrio e seus respectivos autovalores. Modelo reduzido com 3 POMs. $\left(\Gamma_{0}=0,40\right)$.

Tabela 6.5 - Autovalores reais da sela e seus respectivos autovetores. $\left(\Gamma_{0}=0,40\right)$

Tabela 7.1 - Freqüência natural mínima da casca cilíndrica ( $\mathrm{rad} / \mathrm{s})$ para diversas alturas de fluido interno e do tipo de fundo. 


\section{Lista de Símbolos}

\section{Símbolos latinos}

$A_{k}(t), \quad$ amplitude do modo $k$ relativa ao POM $k$.

$\mathrm{A}_{\overline{\mathrm{m}}}(\mathrm{t}), \quad$ amplitude do modo $\overline{\mathrm{m}}$ da expansão modal do fluido

D, rigidez de flexão

E, módulo de elasticidade do material

f função de tensão

$F_{L}, \quad$ pressão lateral harmônica

h, $\quad$ espessura da casca cilíndrica

$\mathrm{H}$, altura do fluido interno

$\mathrm{K}, \quad$ rigidez de membrana

L, $\quad$ comprimento da casca cilíndrica

$\mathrm{R}$, raio da casca cilíndrica

$\mathrm{n}$, número de ondas circunferenciais

$\mathrm{N}_{\mathrm{x}}$, esforço axial

$\mathrm{N}_{\mathrm{x}}^{\mathrm{F}}, \quad$ esforço axial fundamental

$N_{x}^{\prime}, \quad$ esforço axial incremental

$\mathrm{N}_{\theta}$, esforço circunferencial

$N_{\theta}^{F}, \quad$ esforço circunferencial fundamental

$N_{\theta}^{\prime}$, esforço circunferencial incremental

$\mathrm{N}_{\mathrm{x} \theta}$, esforço cisalhante

$\mathrm{N}_{\mathrm{x} \theta}^{\mathrm{F}}, \quad$ esforço cisalhante fundamental

$N_{x \theta}^{\prime}, \quad$ esforço cisalhante incremental

$\mathrm{m}, \quad$ número de semi-ondas longitudinais

$M_{x}$, momento axial

$M_{x}^{F}$, momento axial fundamental

$M_{x}^{l}$, momento axial incremental

$M_{\theta}$, momento circunferencial

$\mathrm{M}_{\theta}^{\mathrm{F}}$, momento circunferencial fundamental

$\mathrm{M}_{\theta}^{\prime}$, momento circunferencial incremental 


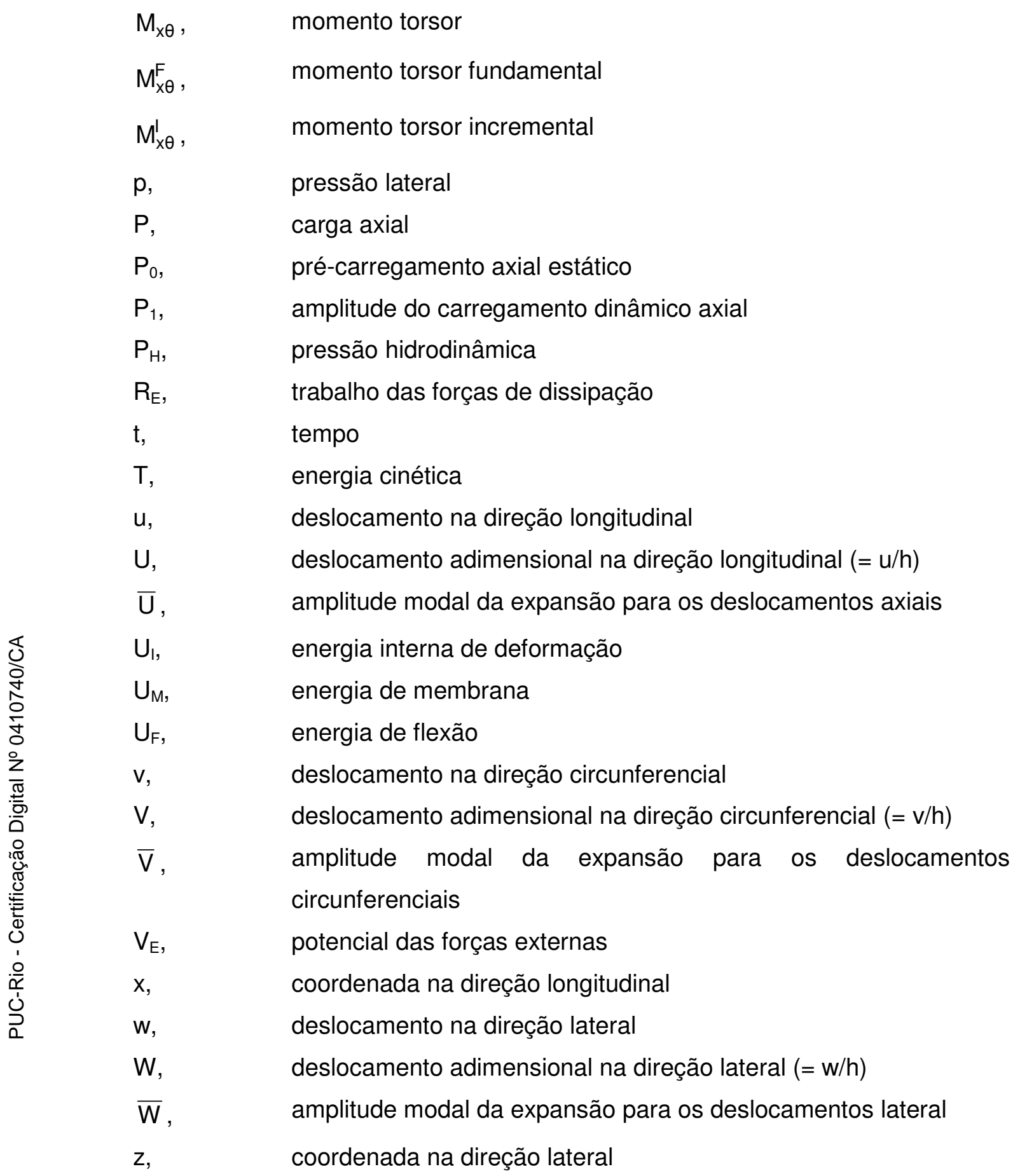

\section{Símbolos gregos}

$\beta_{1}$, amortecimento viscoso

$\beta_{2}, \quad$ amortecimento viscoelástico

$\eta_{1}$, coeficiente de amortecimento viscoso

$\eta_{2}, \quad$ coeficiente de amortecimento viscoelástico

$\delta$, $\quad$ parâmetro adimensional linear da espessura $(=h / R)$ 


\begin{tabular}{|c|c|}
\hline$\Delta$ & parâmetro adimensional quadrático da espessura $\left(=h^{2} / 12 R^{2}\right)$ \\
\hline$\ell$ & parâmetro adimensional do comprimento (= L/R) \\
\hline$\xi$ & coordenada adimensional na direção axial $(=x / R)$ \\
\hline$\phi$ & potencial de velocidade do fluido \\
\hline$\chi_{x}$ & mudança de curvatura axial \\
\hline$\chi_{x}^{F}$ & mudança de curvatura axial fundamental \\
\hline$\chi_{x}^{\prime}$ & mudança de curvatura axial incremental \\
\hline $\bar{\chi}_{x}$ & mudança de curvatura axial de um ponto qualquer da casca \\
\hline$\chi_{\theta}$ & mudança de curvatura circunferencial \\
\hline$\chi_{\theta}^{F}$ & mudança de curvatura circunferencial fundamental \\
\hline$\chi_{\theta}^{\prime}$ & mudança de curvatura circunferencial incremental \\
\hline $\bar{\chi}_{\theta}$ & $\begin{array}{l}\text { mudança de curvatura circunferencial de um ponto qualquer da } \\
\text { casca }\end{array}$ \\
\hline$\chi_{x \theta}$ & mudança de curvatura angular \\
\hline$\chi_{x \theta}^{F}$ & mudança de curvatura angular fundamental \\
\hline$\chi_{x \theta}^{\prime}$ & mudança de curvatura angular incremental \\
\hline $\bar{\chi}_{x \theta}$ & mudança de curvatura angular de um ponto qualquer da casca \\
\hline$\varepsilon_{X}$ & deformação específica axial \\
\hline$\varepsilon_{x}^{F}$ & deformação específica axial fundamental \\
\hline$\varepsilon_{x}^{l}$ & deformação específica axial incremental \\
\hline $\bar{\varepsilon}_{x}$ & deformação específica axial de um ponto qualquer da casca \\
\hline$\varepsilon_{\theta}$ & deformação específica circunferencial \\
\hline$\varepsilon_{\theta}^{F}$ & deformação específica circunferencial fundamental \\
\hline$\varepsilon_{\theta}^{l}$ & deformação específica circunferencial incremental \\
\hline $\bar{\varepsilon}_{\theta}$ & $\begin{array}{l}\text { deformação específica circunferencial de um ponto qualquer da } \\
\text { casca }\end{array}$ \\
\hline$\varepsilon_{k n}$ & raízes da equação de restrição dos efeitos de sloshing \\
\hline$\gamma_{x \theta}$ & deformação específica angular \\
\hline$\gamma_{x \theta}^{F}$ & deformação específica angular fundamental \\
\hline$\gamma_{x \theta}^{\prime}$ & deformação específica angular incremente \\
\hline
\end{tabular}




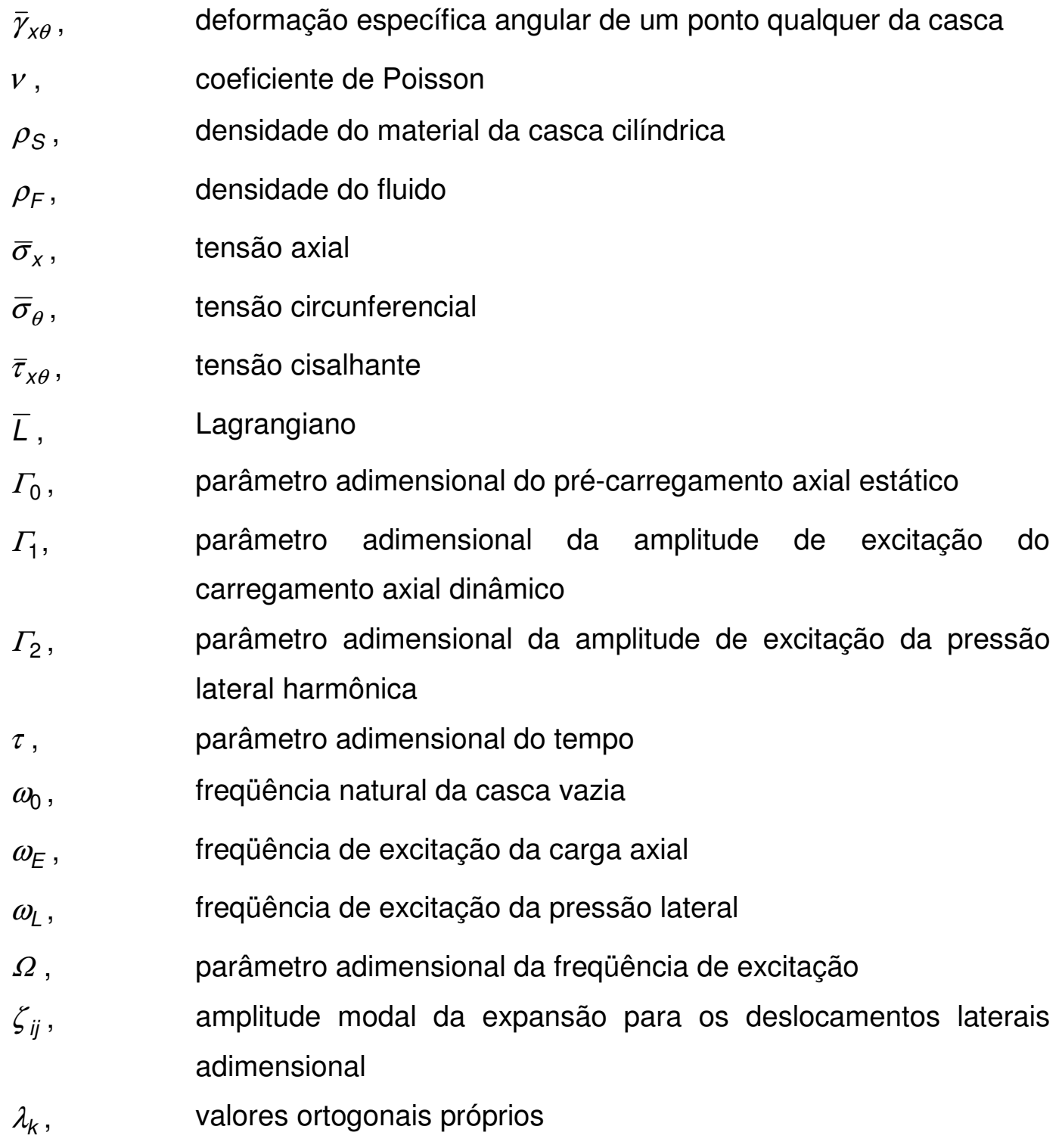

\section{Matrizes e vetores}

$\bar{f}_{k}(\mathbf{x}, \boldsymbol{\theta}), \quad$ modos ortogonais próprios

$\overline{\boldsymbol{\sigma}}, \quad$ vetor de tensões atuantes

$\bar{\varepsilon}, \quad$ vetor de deformações específicas

U, vetor de deslocamentos

$\mathbf{V}(\mathbf{x}, t)$, vetor de distribuição de velocidades do fluido no espaço

$\mathbf{p}(\mathbf{x}, t), \quad$ vetor de distribuição de pressão do fluido no espaço

$\boldsymbol{\rho}(\mathbf{x}, t), \quad$ vetor de distribuição de densidade do fluido no espaço

$\mathbf{w}(\mathbf{x}, \boldsymbol{\theta}, t)$ campo vetorial que descreve o campo deslocamento laterais

C , matriz constitutiva do material 


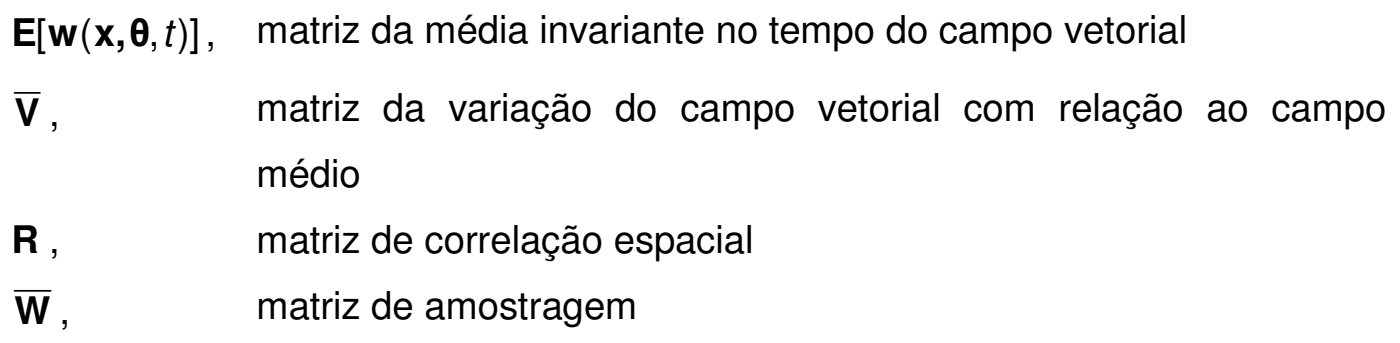

\section{Funções matemáticas}

div, divergente

grad, gradiente

rot, rotacional

$I_{n}$ função modificada de Bessel de primeira classe e ordem $n$

$J_{n}$, função de Bessel de primeira classe e ordem $n$

L( ) operador diferencial linear

$D_{1}($ ) operador diferencial não-linear que gera termos quadráticos

$\mathbf{D}_{2}(\mathrm{l} \quad$ operador diferencial não-linear que gera termos cúbicos 